PROCEEDINGS OF THE

AMERICAN MATHEMATICAL SOCIETY

Volume 132, Number 5, Pages 1321-1331

S 0002-9939(03)07293-9

Article electronically published on December 18, 2003

\title{
AN APPROXIMATION PROPERTY OF HARMONIC FUNCTIONS IN LIPSCHITZ DOMAINS AND SOME OF ITS CONSEQUENCES
}

\author{
JORGE RIVERA-NORIEGA
}

(Communicated by Andreas Seeger)

\begin{abstract}
An extension of an inequality of J. B. Garnett (1979), with improvements by B. E. J. Dahlberg (1980), on an approximation property of harmonic functions is proved. The weighted inequality proved here was suggested by the work of J. Pipher (1993) and it implies an extension of a result of S. Y. A. Chang, J. Wilson and T. Wolff (1985) and C. Sweezy (1991) on exponential square integrability of the boundary values of solutions to second-order linear differential equations in divergence form. This implies a solution of a problem left open by R. Bañuelos and C. N. Moore (1989) on sharp estimates for the area integral of harmonic functions in Lipschitz domains.
\end{abstract}

\section{IntRoduCtion AND STATEMENTS OF THEOREMS}

Consider an operator $L$ of the form

$$
L u=\operatorname{div} A(X) \nabla u(X)
$$

where $A(X)=\left(a_{i, j}(X)\right)$ is an $n \times n$ matrix of measurable functions satisfying the ellipticity condition

$$
\lambda|\xi|^{2} \leq \sum_{i, j} a_{i, j}(X) \xi_{j} \xi_{i} \leq \Lambda|\xi|^{2}
$$

for $\xi \in \mathbb{R}^{n}$. For functions $F: \mathbb{R}_{+}^{n} \longrightarrow \mathbb{R}$ define

$$
B_{\alpha} F(Q)=\left(\int_{\Gamma_{\alpha}(Q)}|\nabla F(X)| \delta(X)^{1-n} d X\right)^{1 / 2},
$$

the nontangential maximal function

$$
N_{\alpha} F(Q)=\sup _{X \in \Gamma_{\alpha}(Q)}|u(X)|
$$

and the area integral operator

$$
S_{\alpha} F(Q)=\left(\int_{\Gamma_{\alpha}(Q)}|\nabla F(X)|^{2} \delta(X)^{2-n} d X\right)^{1 / 2} .
$$

Received by the editors February 26, 2002.

2000 Mathematics Subject Classification. Primary 42B25, 35J67.

Key words and phrases. Approximation of harmonic functions, exponential square class, area integral estimates. 
Here, $\delta(X)=\operatorname{dist}\left(X ; \mathbb{R}^{n-1}\right)$ and $\Gamma_{\alpha}(Q)=\left\{X \in \mathbb{R}_{+}^{n}:|X-Q| \leq(1+\alpha) \delta(X)\right\}$. Also, if $F$ is a solution to $L u=0$, then the gradient of $F$ is defined in the weak sense, and as an $L^{2}$ function, by Cacciopoli's inequality (see e.g. [9] Lemma 1.1.5]). For functions defined in domains $D \subset \mathbb{R}^{n}$ with Lipschitz boundary there is an analogous definition, defining $\delta(X)=\operatorname{dist}(X ; \partial D)$ and $\Gamma_{\alpha}(Q)=\{X \in D: \mid X-$ $Q \mid \leq(1+\alpha) \delta(X)\}$.

The main theorem of this work is the following extension of Garnett-Dahlberg's theorem $([6],[4)$ :

Theorem 1. Let $u$ be a solution to Lu $=0$ on $\mathbb{R}_{+}^{n}$ vanishing at infinity. For every Lipschitz function $\psi: \mathbb{R}^{n-1} \longrightarrow \mathbb{R}$, we define $D(\psi)=\left\{(x, t) \in \mathbb{R}^{n-1} \times \mathbb{R}: t>\right.$ $\psi(x)\}$, and for any $P \in \partial D(\psi)$ and any $r>0$ we define $B_{r}(P)=\left\{X \in \mathbb{R}^{n}\right.$ : $|X-P|<r\}$. Suppose that for some $\tilde{\alpha}>\alpha>0$, any $P \in \partial D(\psi)$ and any $r>0$ we have

$$
\int_{B_{r}(P) \cap \partial D(\psi)}\left[N_{\alpha} u(P)\right]^{2} d \psi(P) \lesssim \int_{B_{r}(P) \cap \partial D(\psi)}\left[S_{\tilde{\alpha}} u(P)\right]^{2} d \psi(P)
$$

(here $d \psi$ denotes the surface measure of $\partial D(\psi)$ ). Then for every $\varepsilon>0$ there exists a function $\varphi: \mathbb{R}^{n-1} \longrightarrow \mathbb{R}$ such that

(i) $\|u-\varphi\|_{L^{\infty}} \leq \varepsilon$,

(ii) For every $C_{0}^{\infty}$ function $w \geq 0$ defined in $\mathbb{R}^{n-1}$, for any cube $\Delta \subset \mathbb{R}^{n-1}$ and with an appropriate aperture $\beta>\alpha$,

$$
\int_{\Delta}\left[B_{\alpha} \varphi(x)\right]^{2} w(x) d x \lesssim \varepsilon \int_{\Delta} w(x) d x+\frac{1}{\varepsilon} \int_{\Delta}\left[S_{\beta} u(x)\right]^{2} w(x) d x .
$$

In the above inequalities, we adopted the standard convention that $A \lesssim B$ means $A \leq c B$, with the constant $c$ depending at most on the ellipticity constants $\lambda, \Lambda$, the dimension $n$, the apertures of the cones, and in some instances the Lipschitz character of a domain $D \subset \mathbb{R}^{n}$. Similarly $A \approx B$ means $A \lesssim B$ and $B \lesssim A$. Also, we will use the notation $w(\Delta)=\int_{\Delta} w(x) d x$.

In [11, it is suggested that a weighted inequality such as (2) would imply a result of [3] on exponential square integrability of the boundary values of harmonic functions in the half plane $\mathbb{R}_{+}^{2}$. We extend this remark to harmonic functions in Lipschitz domains of $\mathbb{R}^{n}$. More precisely, Theorem 1 implies the following.

Theorem 2. Let $D$ be a Lipschitz domain of the form

$$
D=\left\{(x, t) \in \mathbb{R}^{n-1} \times \mathbb{R}: t>\phi(x)\right\},
$$

where $\phi: \mathbb{R}^{n-1} \longrightarrow \mathbb{R}$ is a Lipschitz function, and let $\sigma$ be the surface measure of $\partial D$. Suppose $u$ is a harmonic function in $D$ with boundary values $f$, and with $\left\|S_{\alpha} u\right\|_{L^{\infty}(D)} \equiv\left\|S_{\alpha} u\right\|_{\infty}<\infty$. For any surface cube $\Delta \subset \partial D$, let $f_{\Delta}=\frac{1}{\sigma(\Delta)} \int f d \sigma$. Then there exist constants $c_{1}, c_{2}>0$ independent of the cube $\Delta \subset \partial D$ such that

$$
\frac{1}{\sigma(\Delta)} \int_{\Delta} \exp \left(\frac{c_{1}\left|f(x)-f_{\Delta}\right|^{2}}{\left\|S_{\alpha} u\right\|_{L^{\infty}(D)}^{2}}\right) d \sigma<c_{2}
$$

for all such cubes $\Delta$.

The novelty of our proof is that, unlike the known proofs of related results, we do not reduce matters to the martingale situation to obtain (31), as observed originally in [11]. 
On the other hand, it is well known that exponential square integrability (3) of the boundary values of certain elliptic equations of second order in variational form, implies the following result, posed as an open problem in [2, Problem 4.2.11].

Theorem 3. Let $D$ be a Lipschitz domain as described in Theorem 2 , and let $u$ be a harmonic function in $D$ with boundary values $f$, and with $\left\|S_{\alpha} u\right\|_{\infty}<\infty$. Then, given any surface cube $\Delta$ there exist constants $c_{1}, c_{2}>0$ not depending on $\Delta$ such that

$$
\sigma\left\{P \in \Delta:\left|f(P)-f_{\Delta}\right|>\lambda\right\} \leq c_{1} \exp \left(-\frac{c_{2} \lambda^{2}}{\left\|S_{\alpha} u\right\|_{\infty}^{2}}\right) \sigma(\Delta)
$$

for all $\lambda>0$.

This result implies a sharp control of the nontangential maximal function $N_{\alpha} u$ by the area function $S_{\alpha} u$, for $u$ a harmonic function in $\mathbb{R}_{+}^{n}$, as observed in [2]. Indeed, these authors indicate how to prove such a sharp estimate for arbitrary harmonic functions in two dimensions via a conformal mapping technique, based on the result of [3]. It is also observed in [2, Theorem 3.0.4] that a certain "law of iterated logarithm" for harmonic functions may be obtained from Theorem 2 .

In the next section we prove Theorem 1 and in section 3 we sketch for completeness the arguments to prove Theorems 2, and 3 .

I thank J. Pipher and C. Sweezy for their comments through e-mail communications.

\section{Proof of Theorem 1}

We will construct a function as in [4, Theorem 1] and adapt Dahlberg's arguments to prove the desired properties of this function. At this point we observe that we can mollify this function as in [7, p. 357] to obtain a smooth function satisfying the same properties.

To keep this work self-contained, we include the entire proof.

2.1. We will give the definition of $\varphi$ in the unit cube

$$
\mathcal{U}=\left\{(x, t) \in \mathbb{R}^{n-1} \times \mathbb{R}: 0<x_{i}<1,0 \leq i \leq n-1,0<t<1\right\}
$$

and a dilation and partition of the unit argument will work in the general case. The bottom of this cube is

$$
\mathcal{U}_{0}=\left\{(x, 0): 0<x_{i}<1,0 \leq i \leq n-1\right\} .
$$

We will keep the notation $Q_{0}$ for the bottom of any cube $Q$ in $\mathbb{R}^{n}$ of the form $\prod_{i=1}^{n-1}\left[a_{i}, a_{i}+T\right] \times[0, T], T>0, a_{i} \in \mathbb{R}$.

Now we make a Whitney-type dyadic decomposition of $\mathcal{U}$. Let $\mathcal{F}_{m}$ be the family of dyadic subcubes of $\mathcal{U}_{0}$ of the form $\left[k / 2^{m},(k+1) / 2^{m}\right]^{n-1}$. We will refer to these cubes as dyadic intervals. For $Q \in \mathcal{F}_{m}$, define the rectangles of $\mathcal{U}$ by

$$
\tau(Q)=\left\{(x, t): x \in Q, 1 / 2^{m+1}<t \leq 1 / 2^{m}\right\} .
$$

The family of all rectangles, when varying $m$, will be denoted by $\mathcal{F}$. We will say that $\tau\left(Q_{1}\right)<\tau\left(Q_{2}\right)$ whenever $Q_{1} \subset Q_{2}$, and $Q_{2}$ is in the previous generation of that of $Q_{1}$, i.e., the sidelength of $Q_{2}$ is twice as large as the sidelength of $Q_{1}$. Similarly, if $\tau\left(Q_{1}\right)<\tau\left(Q_{2}\right)$ we say that the latter is the parent of the former, or that the former is the descendant of the latter. $\tau\left(Q_{1}\right)$ and $\tau\left(Q_{2}\right)$ are of the same generation if $Q_{1}$ and $Q_{2}$ have the same sidelength. 
Define for $Z=(z, s)$ and $R \in \mathcal{F}$,

$$
\begin{aligned}
& \Gamma(Z)=\{(x, t):|x-z|<|t-s|\}, \\
& L(R)=\left\{W \in \mathcal{F}: W \cap\left(\bigcup_{P \in R} \Gamma(P)\right) \neq \emptyset\right\} .
\end{aligned}
$$

Notice that, since $\Gamma(Z)$ is a cone with aperture $1, L(R)$ may be viewed as a dyadic conic region of $R$.

2.2. A rectangle $R \in \mathcal{F}$ is called a red rectangle if $\left|\operatorname{osc}_{R} u\right| \geq k_{0} \varepsilon$, for $k_{0}$ a small constant to be determined later. Rectangles that are not red rectangles are called blue rectangles. The definition of oscillation that we adopt here is $\operatorname{osc}_{R} g=\sup _{R} g-$ $\inf _{R} g$.

Let us construct some conic regions formed with blue rectangles. Since the construction will be given inductively and based on a stopping-time, we will focus on the rectangle

$$
S=\left\{(x, t): 1 / 2 \leq t<1,0<x_{i}<1, i=1,2, \ldots, n-1\right\},
$$

and assume that it is a blue rectangle. Associated to $S$, the collection $K(S) \subset \mathcal{F}$ is defined with the following requirements:

(i) $S \in K(S)$,

(ii) $T \in \mathcal{F}$ is added to $K(S)$ if all of the following hold:

- There exists $T^{\prime} \in K(S)$ such that $T<T^{\prime}$.

- All the elements of $L(T)$ are blue intervals.

- For all $\widetilde{T} \in L(T)$, if $P_{W}$ denotes the center of any rectangle $W$, then we have $\left|u\left(P_{S}\right)-u\left(P_{\widetilde{T}}\right)\right|<k_{1} \varepsilon$, with a uniform constant $k_{1}$.

Let $H(S)=\bigcup_{T \in K(S)} L(T)$, and define $D(S)$ as the interior of the closure of $\bigcup_{T \in H(S)} T . D(S)$ is the conic region we were seeking.

Once we have explained how to construct $D(S)$ for the particular choice of $S$, let us go back to our original cube $\mathcal{U}$. Select $S_{1}$ as any blue rectangle of maximal diameter, and obtain with the construction above, mutatis mutandis, the domain $D\left(S_{1}\right)$. If there is a maximal rectangle in $\mathcal{F} \backslash H\left(S_{1}\right)$ that is not a red rectangle, call it $S_{2}$ and repeat inductively the procedure.

Hence, we have obtained a decomposition $\mathcal{U}=\left(\bigcup_{R \in \mathcal{R}} R\right) \cup\left(\bigcup_{j} D\left(S_{j}\right)\right)$, where $\mathcal{R}$ denotes the family of red rectangles. Notice that the sets $R$ and $D\left(S_{j}\right)$ have mutually disjoint interior.

Define

$$
\varphi=\sum_{R \in \mathcal{R}} u \chi(\bar{R})+\sum_{j} u\left(P_{j}\right) \chi\left(D\left(S_{j}\right)\right) \equiv u_{\mathcal{R}}+\sum_{j} u_{j}
$$

$\left(P_{j}=P_{S_{j}}\right.$ is the center of $\left.S_{j}\right)$, where $\chi(W)$ denotes the characteristic function of any set $W \subset \mathbb{R}^{n}$, and $\bar{W}$ denotes its closure.

Clearly $|u(X)-\varphi(X)|<\varepsilon$ for every $X \in \mathcal{U}$. The rest of the proof is devoted to showing that we have the right control over both red rectangles and domains $D\left(S_{j}\right)$.

2.3. In this paragraph we control integrals over the interior of red rectangles.

Lemma 2.3.1. For an appropriate aperture $\beta>\alpha$,

$$
\int_{\mathcal{U}_{0}}\left[B_{\alpha} u_{\mathcal{R}}(x)\right]^{2} w(x) d x \leq \frac{1}{\varepsilon} \int_{\widetilde{\mathcal{U}}_{0}}\left[S_{\beta} u(x)\right]^{2} w(x) d x .
$$


Proof of Lemma. Fix a red rectangle $R \in \mathcal{R}$. We know that there exists a unique dyadic interval $\Delta_{R} \subset \mathcal{U}_{0}$ such that $\tau\left(\Delta_{R}\right)=R$, and that the diameter of $R$ and the sidelength of $\Delta_{R}$ are comparable. Let $\widetilde{\Delta_{R}}$ denote the dilation of $\Delta_{R}$ such that $\Gamma_{\alpha}(P) \cap R \neq \emptyset$ for $P \in \widetilde{\Delta_{R}}$. The $\sim$ notation will be used whenever this dilation occurs for either intervals in $\mathbb{R}^{n-1}$ or rectangles in $\mathbb{R}^{n}$.

We will prove first that

$$
\int_{\Delta_{R}}\left[B_{\alpha} u_{R}(x)\right]^{2} w(x) d x \leq \frac{1}{\varepsilon} \int_{\widetilde{\Delta_{R}}}\left[S_{\beta} u(x)\right]^{2} w(x) d x
$$

where $u_{R}$ is given by $u \chi_{R^{\circ}}$. We have by Fubini's theorem,

$$
\begin{aligned}
\int_{\Delta_{R}}\left[B_{\alpha} u_{R}(x)\right]^{2} w(x) d x & \approx \int_{\mathcal{U}_{0}} \int_{\Gamma_{\alpha}(P)}\left|\nabla u_{R}(X)\right| \delta^{1-n}(X) d X w(P) d P \\
& \lesssim w\left(\widetilde{\Delta_{R}}\right) \int_{R^{\circ}}|\nabla u(X)|^{2} \delta^{1-n}(X) d X .
\end{aligned}
$$

To estimate the right-hand side of this inequality, we observe that by interior estimates of $u$ (see e.g. [10]), and since $\delta(X) \approx \operatorname{diam} R$ for $X \in R$,

$$
\begin{aligned}
k_{0}^{2} \varepsilon^{2} & \leq(\underset{R}{\operatorname{osc} u})^{2} \\
& \lesssim(\operatorname{diam} \widetilde{R})^{2} \frac{1}{|\widetilde{R}|} \int_{\widetilde{R}}|\nabla u(X)|^{2} d X \\
& \lesssim \int_{\widetilde{R}}|\nabla u(X)|^{2} \delta^{2-n}(X) d X \\
& \lesssim \int_{\Gamma_{\beta}(P)}|\nabla u(X)|^{2} \delta^{2-n}(X) d X
\end{aligned}
$$

for $P \in \widetilde{\Delta_{R}}$, when the aperture $\beta$ of the cone is chosen large enough. Multiplying by $w(x)$ and integrating over $\widetilde{\Delta_{R}}$,

$$
w\left(\widetilde{\Delta_{R}}\right) \lesssim \frac{1}{\varepsilon^{2}} \int_{\widetilde{\Delta_{R}}}\left[S_{\beta} u(X)\right]^{2} w(X) d X .
$$

On the other hand, applying Cauchy's inequality, for $P \in \widetilde{\Delta_{R}}$,

$$
\begin{aligned}
\left(\int_{\widetilde{R}^{\circ}}|\nabla u(X)| \delta^{1-n}(X) d X\right)^{2} & \lesssim \int_{\widetilde{R}^{\circ}}|\nabla u(X)|^{2} \delta^{2-n}(X) d X \\
& \lesssim \int_{\Gamma_{\beta}(P)}|\nabla u(X)|^{2} \delta^{2-n}(X) d X
\end{aligned}
$$

where we have used that $\delta(X) \approx \operatorname{diam} R$ for $X \in R$. Once again, multiplying by $w(x)$ and integrating over $\widetilde{\Delta_{R}}$,

$$
\left(\int_{\widetilde{R}^{\circ}}|\nabla u(X)| \delta^{1-n}(X) d X\right)^{2} w\left(\widetilde{\Delta_{R}}\right) \lesssim \int_{\widetilde{\Delta_{R}}} \int_{\Gamma_{\beta}(P)}|\nabla u(X)|^{2} \delta^{2-n}(X) d X w(P) d P
$$

and hence, applying (7) after multiplying by $w\left(\widetilde{\Delta_{R}}\right)$,

$$
w\left(\widetilde{\Delta_{R}}\right) \int_{\widetilde{R}^{\circ}}|\nabla u(X)| \delta^{1-n}(X) d X \lesssim \frac{1}{\varepsilon} \int_{\widetilde{\Delta_{R}}}[S u(P)]^{2} w(P) d P,
$$

which proves (6). 
To obtain an estimate for the sum for $R \in \mathcal{R}$, we define an order relation for red rectangles. Given two red rectangles $R_{1}, R_{2}$, we write $R_{1} \hookrightarrow R_{2}$ whenever $\Delta_{R_{1}} \subseteq \Delta_{R_{2}}$. Let

$$
\mathfrak{R}=\{R \in \mathcal{R}: R \hookrightarrow Q \Longrightarrow Q \text { is a blue rectangle }\}
$$

be the family of maximal red rectangles under the relation $\hookrightarrow$. Then, since the red rectangles are disjoint, we have

$$
\begin{aligned}
\int_{\mathcal{U}_{0}}\left[B_{\alpha} u_{\mathcal{R}}(X)\right]^{2} w(X) d X & \approx \sum_{Q \in \Re} \sum_{R \hookrightarrow Q} w\left(\widetilde{\Delta_{R}}\right) \int_{R^{\circ}}|\nabla u(X)|^{2} \delta^{1-n}(X) d X \\
& \lesssim \sum_{Q \in \Re} \sum_{R \hookrightarrow Q} \frac{1}{\varepsilon} \int_{\widetilde{\Delta_{R}}}\left[S_{\beta} u(P)\right]^{2} w(P) d P \\
& \lesssim \sum_{Q \in \Re} \frac{1}{\varepsilon} \int_{\widetilde{\Delta_{Q}}}\left[S_{\beta} u(P)\right]^{2} w(P) d P \\
& \lesssim \frac{1}{\varepsilon} \int_{\widetilde{\mathcal{U}_{0}}}\left[S_{\beta} u(P)\right]^{2} w(P) d P .
\end{aligned}
$$

Observe that by the same token, using the function $v_{R}=u \chi(\partial R)$, then

$$
\int_{\mathcal{U}_{0}} \sum_{Q \in \Re} \sum_{R \hookrightarrow Q}|\partial R| \delta^{1-n}(X) \chi(\partial R)(X) w(P) d P \lesssim \frac{1}{\varepsilon} \int_{\widetilde{\mathcal{U}}_{0}}\left[S_{\beta} u(P)\right]^{2} w(P) d P .
$$

This implies the lemma.

2.4. Consider now any maximal blue rectangle $S$ and its corresponding $K(S), H(S)$ and $D(S)$. Let $d(S)=\mathcal{U} \cap \partial D(S)$, that is, the part of $\partial D(S)$ inside $\mathcal{U}$, and take $T \in \mathcal{F}$ so that $T \subset \mathcal{U} \backslash D(S)$ and $\partial T \cap d(S) \neq \emptyset$. Construct the chain $T=T_{1}<T_{2}<\cdots<T_{N}=S$, and let $j$ be the smallest integer such that $T_{j} \in K(S)$, in such a way that $T_{j-1} \notin K(S)$. If $L\left(T_{j-1}\right)$ contains a red rectangle $R$, then we will say that $R$ touches $D(S)$, and that $T \in \mathfrak{A}(S)$; otherwise, we say that $T \in \mathfrak{B}(S)$. Notice that if $T$ itself is a red rectangle, then $T \in \mathfrak{A}(S)$. Define

$$
\begin{aligned}
& A(S)=\bigcup_{T \in \mathfrak{A}(S)}(\partial T \cap d(S)), \\
& B(S)=\bigcup_{T \in \mathfrak{B}(S)}(\partial T \cap d(S)) .
\end{aligned}
$$

Let $\theta>1 / 3$ and $L_{j}=\left|\partial D\left(S_{j}\right)\right|$, coming from the decomposition of the paragraph 2.2. According to the previous definitions, we have three types of domains $D\left(S_{j}\right)$ :

$$
\begin{aligned}
I & =\left\{j:\left|\partial D\left(S_{j}\right) \cap \mathcal{U}_{0}\right| \geq \theta L_{j}\right\} \\
I I & =\left\{j:\left|A\left(S_{j}\right)\right| \geq \theta L_{j}\right\} \\
I I I & =\left\{j:\left|B\left(S_{j}\right)\right| \geq \theta L_{j}\right\} .
\end{aligned}
$$

We will prove the estimate

$$
\int_{\mathcal{U}_{0}}\left[B_{\alpha} u_{j}(x)\right]^{2} w(x) d x \lesssim \varepsilon w\left(\widetilde{\mathcal{U}_{0}}\right)+\frac{1}{\varepsilon} \int_{\widetilde{\mathcal{U}_{0}}}\left[S_{\beta} u(x)\right]^{2} w(x) d x
$$

for $j$ in any of the three classes, and in this paragraph we only consider domains belonging to either class $I$ or $I I$. 
For any domain $D\left(S_{j}\right)$ take $T \in \mathcal{F}$ so that $T \subset \mathcal{U} \backslash D\left(S_{j}\right)$ and $\partial T \cap d\left(S_{j}\right) \neq \emptyset$. Let $u_{T}=u \chi_{D_{T}}$, where $D_{T}=\overline{\cup Q}$, and the union is over rectangles $Q \in \mathcal{F}$ such that $Q \subseteq D\left(S_{j}\right)$ and $\bar{Q} \cap \bar{T} \neq \emptyset$. In this case we say that $T$ is adjacent to $D\left(S_{j}\right)$. Observe that $\int_{\Gamma(P)}\left|\nabla u_{T}(X)\right| d X$ may be viewed as the surface measure of $\partial T \cap d\left(S_{j}\right) \cap \Gamma(P)$ multiplied by the size of the jump of the value of $u(X)$ from $d\left(S_{j}\right)$ to any other rectangle, and this jump is of the order of $\varepsilon$.

Note that by Fubini's theorem,

$$
\begin{aligned}
\int_{\Delta_{T}}\left[B_{\alpha}\left(u_{T}\right)(x)\right]^{2} w(x) d x & \lesssim \varepsilon(\operatorname{diam} T)^{1-n}\left|\partial T \cap d\left(S_{j}\right)\right| w\left(\widetilde{\Delta_{T}}\right) \\
& \lesssim \varepsilon(\operatorname{diam} T)^{1-n}(\operatorname{diam} T)^{n-1} w\left(\widetilde{\Delta_{T}}\right)
\end{aligned}
$$

and so

$$
\int_{\Delta_{T}}\left[B_{\alpha}\left(u_{T}\right)(x)\right]^{2} w(x) d x \lesssim \varepsilon w\left(\widetilde{\Delta_{T}}\right)
$$

Domains in class I. Let $T \in \mathcal{F}$ so that $T \subset \mathcal{U} \backslash D\left(S_{j}\right)$ and $\partial T \cap d\left(S_{j}\right) \neq \emptyset$, and let $u_{T}$ be as defined above. Adding over all such rectangles, applying (9), since there is a finite overlapping we obtain

$$
\int_{\mathcal{U}_{0} \cap\left(\cup \widetilde{\Delta}_{T}\right)}\left[B_{\alpha} u_{j}(x)\right]^{2} w(x) d x \lesssim \varepsilon w\left(\widetilde{\mathcal{U}_{0}}\right) .
$$

On the other hand,

$$
\int_{\mathcal{U}_{0} \backslash\left(\cup \widetilde{\Delta_{T}}\right)}\left[B_{\alpha} u_{j}(x)\right]^{2} w(x) d x \lesssim \int_{\Phi}\left[B_{\alpha} u_{j}(x)\right]^{2} w(x) d x=0
$$

since $u_{j}$ is constant in the region

$$
\Phi=\bigcup_{Y \in \mathcal{U}_{0} \backslash\left(\cup \widetilde{\Delta_{T}}\right)} \Gamma(Y) \cap \mathcal{U}
$$

in case this region is not empty. Since the domains are mutually disjoint, we can add them together:

$$
\sum_{j \in I} \int_{\mathcal{U}_{0}}\left[B_{\alpha} u_{j}(x)\right]^{2} w(x) d x \lesssim \varepsilon w\left(\widetilde{\mathcal{U}_{0}}\right)
$$

Domains in class II. Consider now $D\left(S_{j}\right)$ in the class $I I$. The Lipschitz character of $D\left(S_{j}\right)$ and Dahlberg's argument [4, p. 100] imply that

$$
\Delta_{T} \subset \Delta_{R}^{*}
$$

for an appropriate dilation of $\Delta_{R}$, and where $R \subset L(T)$ and $R$ touches $D\left(S_{j}\right)$. Hence

$$
\sum_{T \in \mathfrak{A}\left(S_{j}\right)} w\left(\Delta_{T}\right) \lesssim \sum w\left(\Delta_{R}\right)
$$


where the sum in the right-hand side is taken over the rectangles $R$ that touch $D\left(S_{j}\right)$. Thus, applying (9), (12) and (7),

$$
\begin{aligned}
\int_{\mathcal{U}_{0}}\left[B_{\alpha} u_{j}(x)\right]^{2} w(x) d x & \lesssim \sum_{T} \int_{\Delta_{T}} \int_{\Gamma(P)}\left|\nabla\left(u_{j}\right)_{T}(X)\right| \delta^{1-n}(X) d X w(P) d P \\
& \lesssim \sum_{T} \varepsilon w\left(\Delta_{T}\right) \\
& \lesssim \sum_{R \text { touches } D\left(S_{j}\right)} \varepsilon w\left(\Delta_{R}^{*}\right) \\
& \lesssim \sum_{R \text { touches } D\left(S_{j}\right)} \frac{1}{\varepsilon} \int_{\widetilde{\Delta_{R}}}\left[S_{\beta} u(x)\right]^{2} w(x) d x .
\end{aligned}
$$

Therefore,

$$
\sum_{j \in I I} \int_{\mathcal{U}_{0}}\left[B_{\alpha} u_{j}(x)\right]^{2} w(x) d x \lesssim \sum_{j}\left(\sum_{R \text { touches } D\left(S_{j}\right)} \frac{1}{\varepsilon} \int_{{\widetilde{\Delta_{R}}}_{R}}\left[S_{\beta} u(x)\right]^{2} w(x) d x\right),
$$

and since there is only a finite overlapping of domains $D\left(S_{j}\right)$ that touch a fixed $R$,

$$
\sum_{j \in I I} \int_{\mathcal{U}_{0}}\left[B_{\alpha} u_{j}(x)\right]^{2} w(x) d x \lesssim \frac{1}{\varepsilon} \int_{\widetilde{\mathcal{U}_{0}}}\left[S_{\beta} u(x)\right]^{2} w(x) d x .
$$

2.5. For $j$ in the class $I I I$ we first define the Lipschitz domain

$$
D^{\prime}\left(S_{j}\right)=D\left(S_{j}\right) \backslash \overline{\left(\bigcup_{P \in d\left(S_{j}\right)} \gamma_{P}\right)}
$$

where $\gamma_{P}=\{X:|X|<-\beta t\}, \beta>1$. For $P \in \partial D^{\prime}\left(S_{j}\right)$, we define

$$
M_{j}(P)=\sup \left|u(Q)-u\left(P_{j}\right)\right|,
$$

where the sup is taken over the points $Q$ in the line segment joining $P$ and $P_{j}$, the center of the rectangle $S_{j}$. Also, we define for $P \in \partial D^{\prime}\left(S_{j}\right)$,

$$
N_{j} u(P)=\sup _{X \in \Gamma(P) \cap D^{\prime}\left(S_{j}\right)}|u(X)|
$$

and observe that $M_{S_{j}}(P) \leq 2 N_{j} u(P)$, for all $P \in \partial D^{\prime}\left(S_{j}\right)$. The geometric arguments of [4, p. 101] imply

$$
\left|B\left(S_{j}\right)\right| \lesssim\left|\bigcup_{T \in \mathfrak{B}(S)}\left\{P \in \partial T \cap d\left(S_{j}\right) \cap \partial D^{\prime}\left(S_{j}\right): M_{j} u(P)>\varepsilon / 4\right\}\right|
$$

(here is where we choose $k_{0}$ and $k_{1}$ to define red rectangles and blue regions). 
Applying again (9) and adding over $T \in \mathfrak{B}\left(S_{j}\right)$ with $T \cap \partial D^{\prime}\left(S_{j}\right) \neq \emptyset$, we obtain

$$
\begin{aligned}
\int_{\mathcal{U}_{0}}\left[B_{\alpha} u_{j}(x)\right]^{2} w(x) d x & \approx \sum_{T} \int_{\mathcal{U}_{0}} w(x) \int_{\Gamma(x)}\left|\nabla u_{T}(Y)\right| d^{1-n}(Y) d Y d x \\
& \lesssim \varepsilon \sum_{T} \int_{\Delta_{T}} w(x)\left|\partial T \cap d\left(S_{j}\right)\right| d x(\operatorname{diam} T)^{1-n} \\
& \lesssim \varepsilon \sum_{T} \int_{\Delta_{T}} w(x)\left|\left\{P \in \partial T: M_{j} u(P)>\varepsilon / 4\right\}\right| d x(\operatorname{diam} T)^{1-n} \\
& \lesssim \frac{1}{\varepsilon} \sum_{T} \int_{\Delta_{T}} w(x) \int_{\partial T}\left[N_{j} u(P)\right]^{2} d \sigma_{j}(P) d x(\operatorname{diam} T)^{1-n} .
\end{aligned}
$$

By the assumption (1), and since for $P \in \partial D^{\prime}\left(S_{j}\right)$ there exists $x_{P} \in \mathbb{R}^{n-1}$ such that $\Gamma(P) \subset \Gamma\left(X_{P}\right)$, we continue our estimates as follows:

$$
\begin{aligned}
& \lesssim \frac{1}{\varepsilon} \sum_{T} \int_{\Delta_{T}} w(x)\left(\int_{\partial T} \int_{\Gamma(P)}|\nabla u(Y)|^{2} d^{2-n}(Y) d Y d \sigma_{j}(P)\right) d x(\operatorname{diam} T)^{1-n} \\
& \lesssim \frac{1}{\varepsilon} \sum_{T} \int_{\Delta_{T}} w(x)\left(\int_{\Gamma(x)}|\nabla u(Y)|^{2} d^{2-n}(Y) d Y\right) d x \\
& \lesssim \frac{1}{\varepsilon} \int_{\widetilde{\mathcal{U}}_{0}}[S u(x)]^{2} w(x) d x
\end{aligned}
$$

where we used all the above remarks. We can add over all $j$ in class $I I I$ since these domains are disjoint.

From the estimates in paragraphs 2.3 through 2.5. we may obtain Theorem 1 as in [4, p. 103].

\section{Some consequences of Theorem 1}

For completeness, in this section we include the arguments from [1] to prove Theorem 2. As mentioned before, this extends a theorem from [3] and the main theorem of [13]. We also provide the arguments to prove Theorem 3 .

Define $\Phi: \mathbb{R}_{+}^{n} \longrightarrow D$ by $\Phi(x, y)=(x, y+\phi(x))$. If $u$ is harmonic in $D$, then $v=$ $u \circ \Phi$ is a solution to $L v=0$, for some operator $L$ as described in the introduction, and $\Delta=\Phi(Q)$ for some cube $Q \subset \mathbb{R}^{n}$. Using this map we transfer the situation to $\mathbb{R}_{+}^{n+1}$ and solutions to $L u=0$. Moreover, by the results of [5], the hypothesis (1) holds, and so we may apply Theorem 1 . Also, by the boundedness of $S_{\alpha} u$ we can normalize $u$ so that it vanishes at infinity (cf. [8]).

Given $\Delta \subset \partial D$ an arbitrary surface cube, let $Q \subset \mathbb{R}^{n}$ be the cube such that $\Delta=\Phi(Q)$. Suppose that the sidelength of $Q$ is $r$. To prove Theorem 2 we need to prove

$$
\frac{1}{|Q|} \int_{Q} \exp \left(\frac{c_{1}\left|f(x)-f_{Q}\right|^{2}}{\left\|S_{\alpha} u\right\|_{L^{\infty}\left(\mathbb{R}^{n}\right)}^{2}}\right) d x<c_{2}
$$

with constants independent of $Q$. For $\varepsilon>0$, let $\psi$ be the local approximation to $v$ in a dyadic cube containing $Q$, according to Theorem 1. Fix $x_{0} \in Q$. Observe that for any $x \in Q$,

$$
\psi\left(x_{0}, 0\right)-\psi(x, r)=-\int_{\mathcal{L}} \nabla \psi \cdot d \vec{s},
$$


where $\mathcal{L}$ is the line segment from $\left(x_{0}, 0\right)$ to $(x, r)$. Taking an average over $Q$, and using a change of variables we obtain

$$
\left|\psi\left(x_{0}, 0\right)-\frac{1}{|Q|} \int_{Q} \psi(x, r) d x\right| \leq \int_{\Gamma\left(x_{0}\right)}|\nabla \psi(x, t)| d x \frac{d t}{t^{n-1}} .
$$

Let

$$
c_{Q}=\frac{1}{|Q|} \int_{Q} v(x, r) d x .
$$

Then we have, by the approximation property of $\psi$ and the above inequality,

$$
\begin{aligned}
\left|f\left(x_{0}\right)-c_{Q}\right| \lesssim & \left|f\left(x_{0}\right)-\psi\left(x_{0}, 0\right)\right|+\left|\psi\left(x_{0}, 0\right)-\frac{1}{|Q|} \int_{Q} \psi(y, r) d y\right| \\
& +\left|\frac{1}{|Q|} \int_{Q}[v(y, r)-\psi(y, r)] d y\right| \\
\lesssim & \varepsilon+\int_{\Gamma\left(x_{0}\right)}|\nabla \psi(y, t)| d y \frac{d t}{t^{n-1}} .
\end{aligned}
$$

Multiply by $w$, integrate over $Q$ and apply (2) to obtain

$$
\begin{aligned}
\int_{Q}\left|f(x)-f_{Q}\right| w(x) d x & \lesssim \varepsilon w(Q)+\int_{Q} \int_{\Gamma(x)}|\nabla \psi(y, t)| d y \frac{d t}{t^{n-1}} w(x) d x \\
& \lesssim \varepsilon w(Q)+\frac{1}{\varepsilon} \int_{Q}\left[S_{\alpha} v(x)\right]^{2} w(x) d x \\
& \lesssim \varepsilon \int_{Q} M w(x) d x+\frac{1}{\varepsilon} \int_{Q}\left[S_{\alpha} v(x)\right]^{2} w(x) d x
\end{aligned}
$$

where $M w$ is the Hardy-Littlewood maximal function of $w$. If we now take $w \in L^{q}$ with $\|w\|_{L^{q}} \leq 1,1 / q+1 / p=1,1<q<2$, then we get

$$
\frac{1}{|Q|} \int_{Q}\left|f-f_{Q}\right|^{p} \lesssim \frac{1}{\varepsilon}\left\|S_{\alpha} v\right\|_{\infty}^{2}+\varepsilon p
$$

by a well-known estimate for the maximal function [12, p. 14]. Choosing $\varepsilon=$ $\left\|S_{\alpha} v\right\|_{\infty} / \sqrt{p}$ we obtain, for $c_{1}=\delta>0$ small,

$$
\begin{aligned}
\frac{1}{|Q|} \int_{Q} \exp \left(\frac{\delta\left|f(x)-f_{Q}\right|^{2}}{\left\|S_{\alpha} v\right\|_{\infty}^{2}}\right) d x & \leq \sum_{k} \frac{\delta^{k}}{k !} \frac{1}{|Q|} \int_{Q}\left(\frac{\left|f(x)-f_{Q}\right|^{2}}{\left\|S_{\alpha} v\right\|_{\infty}^{2}}\right)^{2 k} d x \\
& \leq \sum_{k} \frac{\delta^{k}}{k !}(C)^{2 k} \cdot k^{k}<\infty
\end{aligned}
$$

This proves (15) and hence Theorem 2, after some applications of the mapping $\Phi$ to transfer the result to the domain $D$. 
Observe that by Chebyshev's inequality and (15),

$$
\begin{aligned}
\left|\left\{x \in Q:\left|f(x)-f_{Q}\right|>\lambda\right\}\right| & =\mid\left\{x \in Q: \exp \left(\frac{c_{1}\left|f(x)-f_{Q}\right|^{2}}{\left\|S_{\alpha} v\right\|_{\infty}^{2}}\right)\right. \\
& \left.>\exp \left(\frac{c_{1} \lambda^{2}}{\left\|S_{\alpha} v\right\|_{\infty}^{2}}\right)\right\} \mid \\
& \leq \exp \left(-\frac{c_{1} \lambda^{2}}{\left\|S_{\alpha} v\right\|_{\infty}^{2}}\right) \int_{Q} \exp \left(\frac{c_{1}\left|f(x)-f_{Q}\right|^{2}}{\left\|S_{\alpha} v\right\|_{\infty}^{2}}\right) d x \\
& \leq c_{2}|Q| \exp \left(-\frac{c_{1} \lambda^{2}}{\left\|S_{\alpha} v\right\|_{\infty}^{2}}\right),
\end{aligned}
$$

which proves Theorem [3, after transfering these estimates to $D$ via $\Phi$.

\section{REFERENCES}

[1] R. Bañuelos and C. N. Moore, Sharp estimates for the nontangential maximal function and the Lusin area function in Lipschitz domains, Trans. Amer. Math. Soc. 312 (1989), 641-662. MR 90i:42030

[2] R. Bañuelos and C. N. Moore, Probabilistic behavior of harmonic functions, Progress in Mathematics, vol. 175, Birkhäuser-Verlag, Basel-Boston-Berlin, 1999. MR 2001j:31003

[3] S. Y. A. Chang, J. M. Wilson, and T. H. Wolff, Some weighted norm inequalities concerning the Schrödinger operators, Comment. Math. Helvetici 60 (1985), 217-246. MR 87d:42027

[4] B. E. J. Dahlberg, Approximation of harmonic functions, Ann. Inst. Fourier Grenoble 30 (1980), 97-107. MR 82i:31010

[5] B. E. J. Dahlberg, Weighted norm inequalities for the Lusin area integral and the nontangential maximal functions for functions harmonic in a Lipschitz domain, Studia Math. 67 (1980), 297-314. MR 82f:31003

[6] J. B. Garnett, Two constructions in BMO, Proc. Sympos. Pure Math., Vol. 35, Amer. Math. Soc., Providence, RI, 1979, pp. 295-301. MR 81d:30058

[7] J. B. Garnett, Bounded analytic functions, Pure and Applied Math., Vol. 96, Academic Press, New York, 1981. MR 83g:30037

[8] R. Gundy and R. Wheeden, Weighted integral inequalities for the nontangential maximal functions, Lusin area integral, and Walsh-Paley series, Studia Math. 49 (1974), 107-124. MR 50:5340

[9] C. E. Kenig, Harmonic analysis techniques for second order elliptic boundary value problems, CBMS Regional Conference Series in Mathematics, no. 83, Amer. Math. Soc., Providence, RI, 1994. MR 96a:35040

[10] J. Moser, On Harnack's theorem for elliptic differential equations, Comm. Pure and Appl. Math. 14 (1961), 577-591. MR 28:2356

[11] J. Pipher, A martingale inequality related to exponential square integrability, Proc. Amer. Math. Soc. 118 (1993), 541-546. MR 94c:42015

[12] E. M. Stein, Harmonic analysis: Real-variable methods, orthogonality, and oscillatory integrals, Princeton Univ. Press., Princeton, NJ, 1993. MR 95c:42002

[13] C. Sweezy, L-harmonic functions and the exponential square class, Pacific J. of Math. 147 (1991), 187-200. MR 91k:35072

Department of Mathematics, University of Illinois, Urbana, Illinois 61801

E-mail address: rnoriega@math.uiuc.edu 